\title{
Reconstructing the exit wave in high-resolution transmission electron microscopy using machine learning
}

Jakob Schiøtz ${ }^{1}$, Frederik Dahl ${ }^{2}$, Matthew Helmi Leth Larsen ${ }^{1}$, Christian Kisielowski ${ }^{3}$, Stig Helveg ${ }^{4}$, Ole Winther ${ }^{5}$ and Thomas Hansen ${ }^{6}$

${ }^{1}$ Department of Physics, Technical University of Denmark, Kongens Lyngby, Hovedstaden, Denmark, ${ }^{2}$ Department of Physics, Technical University of Denmark, Hovedstaden, Denmark, ${ }^{3}$ Lawrene Berkeley National Laboratory, The Molecular Foundry and Joint center for Artifical Photosynthesis, University of California, Berkeley, United States, ${ }^{4}$ Center for Visualizing Catalytic Processes (VISION), Department of Physics, Technical University of Denmark, DK-2800 Kgs. Lyngby, Denmark., Kongens Lyngby, Hovedstaden, Denmark, ${ }^{5}$ Department of Applied Mathematics and Computer Science, Technical University of Denmark, Kongens Lyngby, Hovedstaden, Denmark, ${ }^{6}$ DTU Nanolab, Technical University of Denmark, Kgs. Lyngby, Hovedstaden, Denmark

High-resolution Transmission Electron Microscopy (HRTEM) has become a powerful technique for examining matter at the atomic-scale. In particular the ability to acquire single projection images of the sample volume in a parallel fashion while employing low electron doses and dose-rates makes HRTEM attractive to detect the atomic-scale realm of matter [1]. However, interpreting the image is not always straightforward, as the image is formed by phase contrast.

A focal series of HRTEM images enables reconstruction of the exit wave function, which provides the most informative measure of the sample. A series of typically around 20-50 images with varying defocus is used to numerically reconstruct the most likely exit wave, which can then be interpreted as it corresponds closely to the structure of the sample $[2,3]$.

We here show that a convolutional neural network is able to reconstruct the exit wave function from a focal series of only two or three images - but not from a single image. The convolutional neural network is based on the U-Net architecture [4] and is almost the same as the one we have previously used to identify the positions of atomic columns in single images of metallic nanoparticles [5].

We train the neural networks on simulated images. The simulated images are produced with the multislice algorithm, both the exit wave function and images produced with three different values of the defocus are saved. We use either the pyqstem[6] or the abtem[7] software for the simulations. The exit waves are then blurred slightly by folding with a gaussian, and a neural network is trained to reconstruct the exit wave from the images. The network is validated on a different set of simulated images, and if applicable applied to experimentally obtained data.

In all cases we work with thin flakes of 2D materials, although the methods should generalize to other classes of materials. We train and validate networks based on three different types of samples, of increasing difficulty. The first two are unsupported nanoflakes of molybdenum disulphide $\left(\mathrm{MoS}_{2}\right)$, and molybdenum disulphide supported on a graphene substrate (figure 1). $\mathrm{MoS}_{2}$ is an active catalyst for photooxidation, hydrogen evolution and hydrodesulphurization reactions. The third type is a diverse collection of existing and proposed quasi-two-dimensional materials, taken from the Computational 2D Materials Database (C2DB) [8]. In all cases the neural network is able to learn to reconstruct the exit wave function of the vast majority of the samples in the validation sets (see figures 1 and 2), although the C2DB contain some materials with complex structures with overlapping atomic positions, where the network makes errors reconstructing the exit wave. 
Finally, we demonstrate that the network trained on simulated data for graphene-supported molybdenum disulphide can also be used to analyze images from an experimental focus series taken on a sample of a realistic model of an industrial MoS2 based hydrodesulphurization catalyst. The neural network is able to reconstruct the exit wave with such fidelity that the atomic structure of both the MoS2 nanoparticle and the supporting graphene is clearly recovered (to be published).
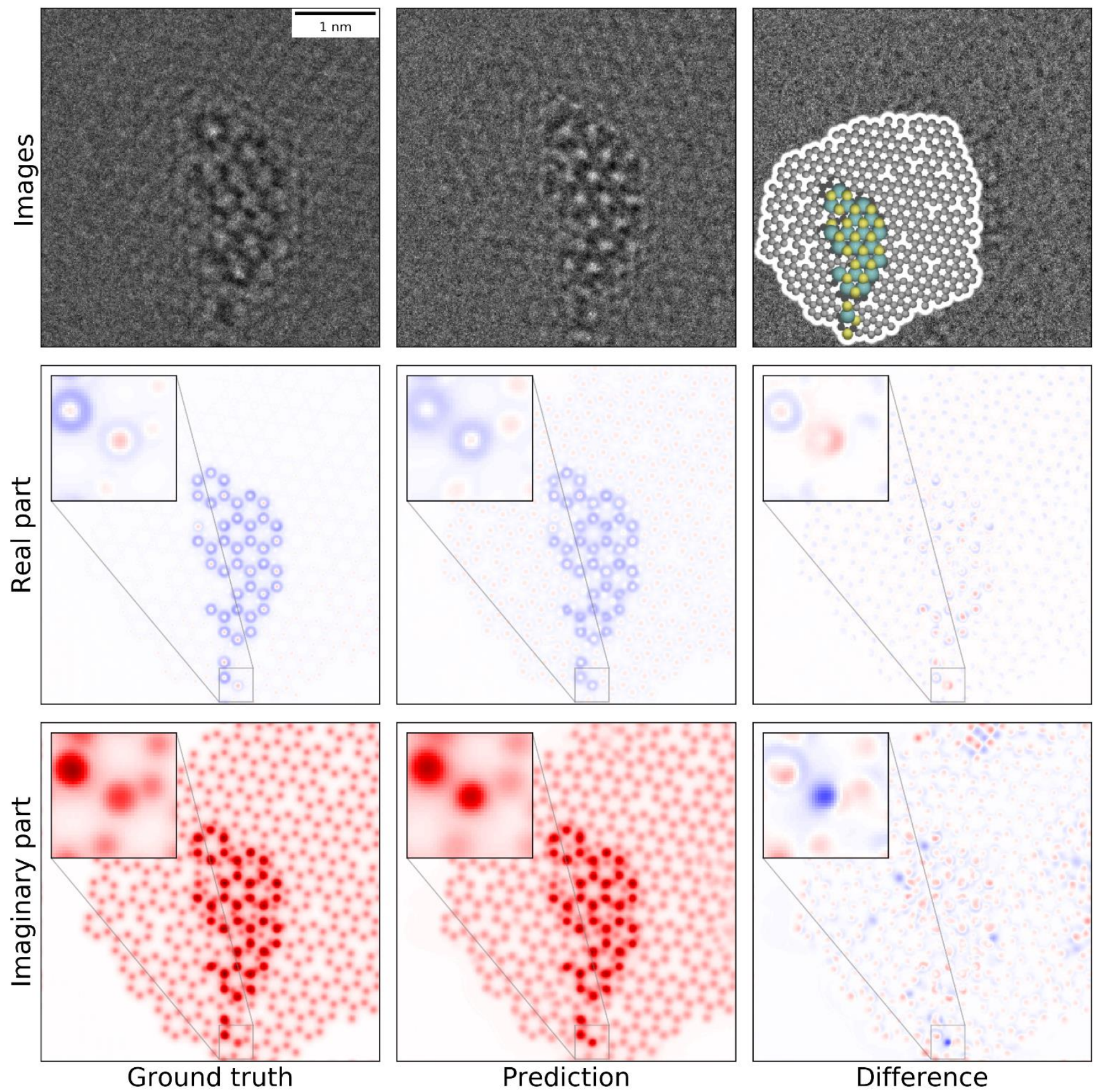

\section{Difference}

Figure 1. Reconstruction of the exit-wave of a MoS2 nanoparticle supported on graphene, by a network trained on a mixture of supported and unsupported MoS2 nanoparticles. The first row shows the three simulated HRTEM figures with varying defocus, the atomic structure is shown as an overlay on the third. The middle and lower row show the real and imaginary parts of the exit-wave, with the first column showing the actual wave function (the ground truth), the second showing the exit-wave reconstructed by the neural network, and 
the last showing the difference. The inset highlights the point with the largest deviation, it is a sulphur vacancy (i.e. a single $\mathrm{S}$ atom instead of a dimer) where the network incorrectly assigns an exit wave which is intermediate between a sulphur vacancy and a complete sulphur dimer. Other sulphur vacancies are detected correctly.
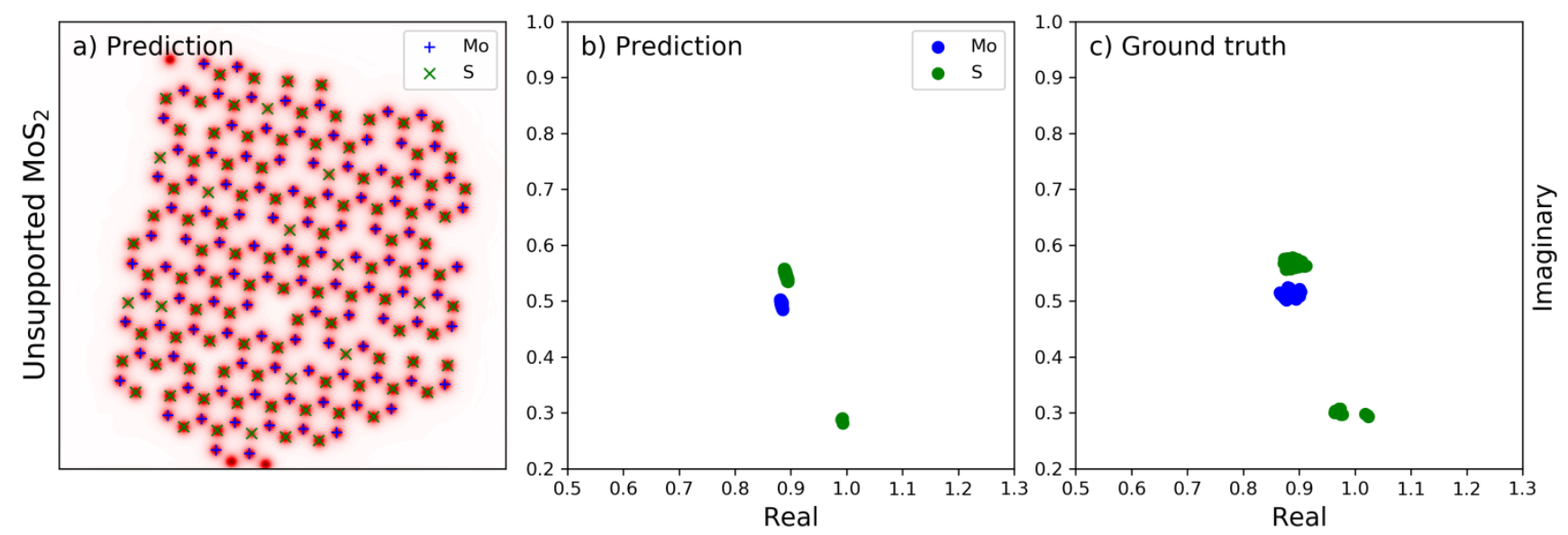

Figure 2. Argand plot of the complex value of the exit wave function at the local maxima of the change in wave function for an unsupported MoS2 nanoparticle. a) The imaginary part of the reconstructed wave function. The peaks are marked with green or blue crosses depending on whether they correspond to Mo or $\mathrm{S}$ atoms. b) Argand plot of the reconstructed wave function. We see a small separation between Mo atoms and $\mathrm{S}$ dimers, a more realistic modelling of atomic vibrations will alter this separation, and may cause the Mo and $\mathrm{S}$ spots to change places in the Argand plot. The green points at the bottom correspond to $\mathrm{S}$ vacancies, where a column contains a single $S$ atom instead of two. c) The Argand plot of the ground truth exit wave, here the $\mathrm{S}$ vacancies separate in two groups depending on whether it is the top or bottom $\mathrm{S}$ atom that is missing, this detail is not captured by the neural network.

\section{References}

1. Kisielowski, C., Frei, H., Specht, P., Sharp, I. D., Haber, J. A., Helveg, S., Adv. Struct. Chem. Imag. 2, 13 (2016). doi:10.1186/s40679-016-0027-9

2. de Beeck, M.O., Van Dyck, D., Coene, W., Ultramicroscopy 64, 167-183 (1996). doi:10.1016/03043991(96)00058-7

3. Tiemeijer, P.C., Bischoff, M., Freitag, B., Kisielowski, C., Ultramicroscopy 118, 35-43 (2012). doi:10.1016/j.ultramic.2012.03.019

4. Ronneberger, O., Fischer, P., Brox, T., in: Medical Image Computing and Computer-Assisted Intervention - MICCAI 2015, pp. 234-241. Springer, Cham (2015). doi:10.1007/978-3-319-24574-4_28

5. Madsen, J., Liu, P., Kling, J., Wagner, J.B., Hansen, T.W., Winther, O., Schiøtz, J., Adv. Theory Simul. 1, 1800037 (2018). doi:10.1002/adts.201800037

6. Madsen, J., Liu, P., Wagner, J. B., Hansen, T. W., Schiøtz, J., Adv. Struct. Chem. Imag. 3, 14 (2017). doi:10.1186/s40679-017-0047-0

7. Madsen, J. and Susi, T., Microsc. Microanal. 26 (Suppl. 2), 448-450 (2020). doi:10.1017/S1431927620014701

8. Haastrup, S. et al., 2D Materials 5, 042002 (2018). doi:10.1088/2053-1583/aacfc1 See also https://cmr.fysik.dtu.dk/c2db/c2db.html 